\title{
Predicting Neutrinoless Double Beta Decay
}

\author{
M. Hirsch A Aillanova del Moral f and J. W. F. Valle团 \\ AHEP Group, Instituto de Física Corpuscular - C.S.I.C./Universitat de València \\ Edificio Institutos de Paterna, Apt 22085, E-46071 Valencia, Spain
}

Ernest $\mathrm{Ma} \S$

Physics Department, University of California, Riverside, CA 92521, USA, and

Institute for Particle Physics Phenomenology, University of Durham, Durham, DH1 3LE, UK

(Dated: November 21, 2018)

\begin{abstract}
We give predictions for the neutrinoless double beta decay rate in a simple variant of the $A_{4}$ family symmetry model. We show that there is a lower bound for the $\beta \beta_{0 \nu}$ amplitude even in the case of normal hierarchical neutrino masses, corresponding to an effective mass parameter $\left|m_{e e}\right| \geq$ $0.17 \sqrt{\Delta m_{\mathrm{ATM}}^{2}}$. This result holds both for the CP conserving and CP violating cases. In the latter case we show explicitly that the lower bound on $\left|m_{e e}\right|$ is sensitive to the value of the Majorana phase. We conclude therefore that in our scheme, $\beta \beta_{0 \nu}$ may be accessible to the next generation of high sensitivity experiments.
\end{abstract}

PACS numbers: 11.30.Hv, 14.60.Pq, 12.60.Fr, 12.60.-i, 23.40.-s

Are neutrinos their own antiparticles? This is one of the most basic current unknowns in neutrino physics. Of all possible manifestations of neutrino masses, neutrinoless double beta decay ( $\beta \beta_{0 \nu}$, for short) offers - to date - the only potentially viable way to answer this question. If $\beta \beta_{0 \nu}$ exists, then neutrino masses are Majorana in nature, irrespective of their ultimate origin [1]. Together with cosmology [2] and direct kinematical searches in tritium decay [3], $\beta \beta_{0 \nu}$ offers one of the three main complementary ways to probe the absolute scale of neutrino masses.

Current experimental limits from $\beta \beta_{0 \nu}$ on the effective Majorana mass of the neutrino $m_{e e}$ are of order $m_{e e} \leq 0.3-1 \mathrm{eV}$ [4, 5]. We note that a claim for a finite $\beta \beta_{0 \nu}$ rate has been published in 6 , but this has so far not been confirmed by any other experiment. A recent proposal 7] aims explicitly at testing the halflife range suggested in [6]. However, future $\beta \beta_{0 \nu}$ experiments may be able to reach down to much lower mass scales. Many experiments sensitive to $m_{e e} \simeq 0.05 \mathrm{eV}$ have already been discussed; see for example 8, 9]. In the longer-term future, even $m_{e e} \simeq 0.01 \mathrm{eV}$ [10, 11] does not seem impossible.

The historic confirmation of neutrino oscillations over the last few years [12], together with some basic theory, suggests that $\beta \beta_{0 \nu}$ is expected, although in general no lower bound on the magnitude of the expected effect can be given. Theoretical input is therefore needed. Currently the origin of neutrino masses is completely unknown. The basic dimension-five operator which leads to neutrino masses [13] can arise from a variety of mech-

\footnotetext{
*Electronic address: Martin.Hirsch@ific.uv.es

${ }^{\dagger}$ Electronic address: Albert.Villanova@ific.uv.es

‡Electronic address: valle@ific.uv.es

$\S$ Electronic address: ma@phyun8.ucr.edu
}

anisms characterized by vastly different scales. Alternatives include the seesaw mechanism [14, 15, 16] and low-energy $R$-parity violating supersymmetry [17]. In neither case is it possible, in general, to establish a lower bound on the magnitude of the $\beta \beta_{0 \nu}$ rate.

Here we consider a simple phenomenological model based on a new realization of the $A_{4}$ family symmetry $[18,19,20]$ in which no $\mathrm{SU}(3) \otimes \mathrm{SU}(2) \otimes \mathrm{U}(1)$ singlet neutrinos are introduced. Instead, the small neutrino masses arise from the small induced vacuum expectation values $(\mathrm{VEVs})$ generated for the neutral components of triplet Higgs bosons [15, 21], transforming nontrivially under the $A_{4}$ family symmetry. The lepton and Higgs particle content and their transformation properties un$\operatorname{der} A_{4}$ and $\mathrm{SU}(2) \otimes \mathrm{U}(1)$ are specified in Table I. With

\begin{tabular}{|c||c||c||c|c|c||c|c|c||c|}
\hline Fields & $L$ & $l^{c}$ & $\phi_{1}$ & $\phi_{2}$ & $\phi_{3}$ & $\eta_{1}$ & $\eta_{2}$ & $\eta_{3}$ & $\xi$ \\
\hline \hline$A_{4}$ & $\mathbf{3}$ & $\mathbf{3}$ & $\mathbf{1}$ & $\mathbf{1}^{\prime}$ & $\mathbf{1}^{\prime \prime}$ & $\mathbf{1}$ & $\mathbf{1}^{\prime}$ & $\mathbf{1}^{\prime \prime}$ & $\mathbf{3}$ \\
\hline \hline$S U(2)_{L}$ & $\mathbf{2}$ & $\mathbf{1}$ & \multicolumn{2}{|||}{$\mathbf{2}$} & \multicolumn{3}{|c||}{$\mathbf{3}$} & $\mathbf{3}$ \\
\hline$Y$ & -1 & 2 & \multicolumn{3}{|c||}{-1} & \multicolumn{2}{|c|}{2} & 2 \\
\hline
\end{tabular}

TABLE I: Lepton and scalar boson quantum numbers

these transformation properties, the charged lepton mass matrix is already diagonal in the flavor basis, with

$$
\begin{aligned}
& m_{e}=h_{1} v_{1}+h_{2} v_{2}+h_{3} v_{3} \\
& m_{\mu}=h_{1} v_{1}+\omega h_{2} v_{2}+\omega^{2} h_{3} v_{3} \\
& m_{\tau}=h_{1} v_{1}+\omega^{2} h_{2} v_{2}+\omega h_{3} v_{3}
\end{aligned}
$$

where $h_{i}$ are charged lepton Yukawa couplings, $v_{i}=\left\langle\phi_{i}^{0}\right\rangle$ and $\omega$ is a complex cubic root of unity satisfying $1+\omega+$ $\omega^{2}=0$. The neutrino mass matrix is then of the form

$$
M_{\nu}=\left(\begin{array}{ccc}
a+b+c & f & e \\
f & a+\omega b+\omega^{2} c & d \\
e & d & a+\omega^{2} b+\omega c
\end{array}\right)
$$


where the only non-diagonal entries are those of the $A_{4}$ triplet $\xi$. Here we have defined

$$
\begin{array}{ll}
a=\lambda_{1}\left\langle\eta_{1}^{0}\right\rangle & d=\kappa\left\langle\xi_{1}^{0}\right\rangle \\
b=\lambda_{2}\left\langle\eta_{2}^{0}\right\rangle & e=\kappa\left\langle\xi_{2}^{0}\right\rangle \\
c=\lambda_{3}\left\langle\eta_{3}^{0}\right\rangle & f=\kappa\left\langle\xi_{3}^{0}\right\rangle
\end{array}
$$

where $\lambda_{i}, \kappa$ and $\left\langle\eta_{i}^{0}\right\rangle,\left\langle\xi_{i}^{0}\right\rangle$ are triplet Yukawa couplings, and VEVs, respectively. Let us further assume that the conditions $b=c$, and $d=e=f$ hold. Whereas the former is an ad hoc assumption, the latter can be maintained naturally because of a residual $Z_{3}$ symmetry. Note that $M_{\nu}$ has a very remarkable (and possibly unique) property here in that each entry is renormalized by the charged-lepton Yukawa couplings in the same way, i.e. $\left|h_{1}\right|^{2}+\left|h_{2}\right|^{2}+\left|h_{3}\right|^{2}$, instead of being proportional to $m_{i}^{2}+m_{j}^{2}$ as in the Standard Model.

It is easy to see that in the above limit we have the prediction

$$
\theta_{23}=\pi / 4, \quad \theta_{13}=0
$$

which matches well with the neutrino oscillation data [12]. Furthermore it can be shown that, in the limit where the solar mass splitting is neglected, $b$ and $d$ can be made real, so that the atmospheric neutrino mass splitting takes on a very simple form

$$
\Delta m_{32}^{2}=6 b d \equiv \Delta m_{\mathrm{ATM}}^{2}
$$

The solar neutrino mass splitting $\Delta m_{\mathrm{SOL}}^{2} \ll \Delta m_{\mathrm{ATM}}^{2}$ can be expressed as

$$
\Delta m_{21}^{2}=\sqrt{T_{1}^{2}+T_{2}^{2}+T_{3}^{2}} \equiv \Delta m_{\mathrm{SOL}}^{2}
$$

where

$$
\begin{aligned}
T_{1} & \equiv 6 \sqrt{2}|b||d| \sin \left(\phi_{2}\right) \\
T_{2} & \equiv 2 \sqrt{2}|d|\left(2|a| \cos \left(\phi_{1}\right)+|b| \cos \left(\phi_{2}\right)+|d|\right) \\
T_{3} & =-3|b|^{2}+|d|^{2}-6|a||b| \cos \left(\phi_{1}+\phi_{2}\right) \\
& +2|a||d| \cos \left(\phi_{1}\right)-2|b||d| \cos \left(\phi_{2}\right)
\end{aligned}
$$

with $\phi_{1} \equiv \phi_{a}-\phi_{d}$ and $\phi_{2} \equiv \phi_{d}-\phi_{b}$, where $a=$ $|a| \exp \left(i \phi_{a}\right), b=|b| \exp \left(i \phi_{b}\right)$ and $d=|d| \exp \left(i \phi_{d}\right)$. The condition in Eq. (4) leads to three inequalities

$$
\left|T_{i}\right| \leq \Delta m_{\mathrm{SOL}}^{2}
$$

which, normalized by $\Delta m_{\text {ATM }}^{2}$, can be expressed in terms of the small parameter $\alpha \equiv \Delta m_{\mathrm{SOL}}^{2} / \Delta m_{\mathrm{ATM}}^{2}$ as

$$
\begin{gathered}
\sqrt{2}\left|\sin \left(\phi_{2}\right)\right| \leq \alpha \\
\frac{\sqrt{2}}{3|b|}|2| a\left|\cos \left(\phi_{1}\right)+\right| b\left|\cos \left(\phi_{2}\right)+\right| d|| \leq \alpha
\end{gathered}
$$

$$
\begin{array}{r}
\left.\frac{1}{6|b||d|}|-3| b\right|^{2}+|d|^{2}-6|a||b| \cos \left(\phi_{1}+\phi_{2}\right) \\
+2|a||d| \cos \left(\phi_{1}\right)-2|b||d| \cos \left(\phi_{2}\right) \mid \leq \alpha
\end{array}
$$

where the current allowed values of $\alpha$ are shown in Fig.13 of Ref. [12].

As for the solar mixing angle, it is given here by

$$
t_{2 S} \equiv \tan \left(2 \theta_{12}\right)=\frac{2 \sqrt{2} d}{3 b-d}
$$

which reduces to

$$
\tan ^{2} \theta_{12}=1 / 2
$$

in two ways, namely,

$$
b=0, \quad b=2 d / 3
$$

Current fits of solar, reactor, atmospheric and accelerator neutrino oscillation data lead to a best fit point to the solar mixing angle for which $\tan ^{2} \theta_{12}$ is slightly less than $1 / 2$. Performing a series expansion of $\tan ^{2} \theta_{12}$ around the two solutions in Eq. (13), we get

$$
\begin{aligned}
\tan ^{2} \theta_{12} & \simeq \frac{1}{2}+\frac{b}{d} \\
\tan ^{2} \theta_{12} & \simeq \frac{1}{2}-\frac{1}{d}\left(b-\frac{2}{3} d\right)
\end{aligned}
$$

These two branches are depicted in Fig. 11 The positive

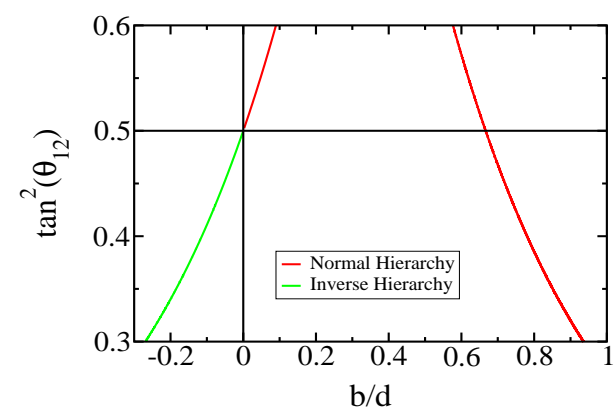

FIG. 1: Solar mixing angle, $\tan ^{2} \theta_{12}$, vs. $b / d$.

horizontal axis corresponds to a normal hierarchy $(\mathrm{NH})$ neutrino spectrum, while the negative part corresponds to inverse hierarchy (IH), as depicted in Fig. 1] This behavior is also recognized in the model of [22].

We now turn to neutrinoless double beta decay. The neutrino-exchange amplitude for this process is simply given by

$$
\left\langle m_{\nu}\right\rangle=m_{e e}=a+2 b
$$

In the case of real parameters, we can solve the following 
system of equations

$$
\begin{gathered}
\Delta m_{\mathrm{SOL}}^{2} \equiv \Delta m_{21}^{2}=|2 a+b+d| \sqrt{(d-3 b)^{2}+8 d^{2}} \\
\Delta m_{\mathrm{ATM}}^{2} \equiv \Delta m_{32}^{2}=6 b d \\
t_{2 S} \equiv \tan \left(2 \theta_{12}\right)=\frac{2 \sqrt{2} d}{3 b-d}
\end{gathered}
$$

and express the parameters $a, b$ and $d$ in terms of experimentally measurable ones $\Delta m_{\mathrm{SOL}}^{2}, \Delta m_{\mathrm{ATM}}^{2}$ and $t_{2 S}$. Substituting in Eq. (16) we can therefore express $\left\langle m_{\nu}\right\rangle$ in terms of these measured observables. We then obtain, up to an overall sign,

$$
\begin{aligned}
& \frac{m_{e e}}{\sqrt{\Delta m_{\mathrm{ATM}}^{2}}}=\operatorname{Sign}\left[\Delta m_{\mathrm{ATM}}^{2}\right] \frac{1}{\sqrt{2 \sqrt{2} t_{2 S}+t_{2 S}^{2}}} \\
& \pm \operatorname{Sign}\left[2 \sqrt{2} t_{2 S}+t_{2 S}^{2}\right] \frac{\alpha \sqrt{2 \sqrt{2} t_{2 S}+t_{2 S}^{2}}}{4 \sqrt{1+t_{2 S}^{2}}}
\end{aligned}
$$

The calculated values of $\left|m_{e e} / \sqrt{\Delta m_{\text {ATM }}^{2}}\right|$ as functions of $t_{2 S}$ according to Eq. (17) are shown in Fig. 2 It can

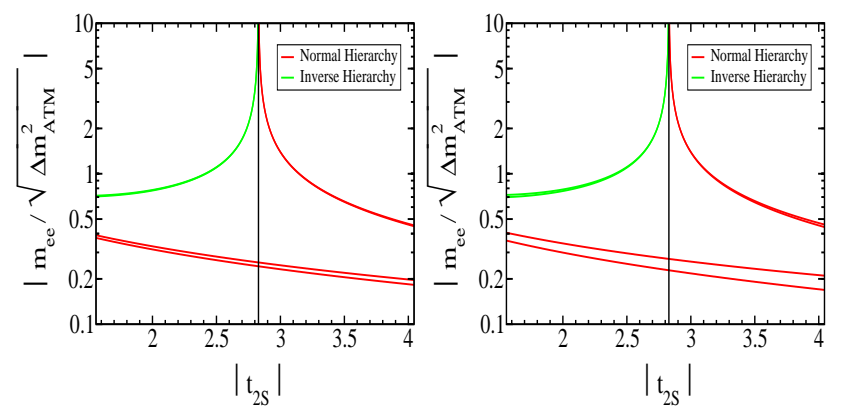

FIG. 2: $\left|m_{e e} / \sqrt{\Delta m_{\text {AтM }}^{2}}\right|$ vs. $\left|t_{2 S}\right|$ according to Eq. (17). The bound depends slightly on the value of $\alpha$ : the left panel corresponds to $\alpha=0.022$ and the right one to $\alpha=0.065$. The dark (red) lines correspond to normal hierarchy, while the grey (green) line is for the inverse hierarchy case. The vertical line corresponds to the horizontal line in Fig. 11 at $\tan ^{2} \theta_{12}=1 / 2$.

be seen that given the currently allowed experimental $3 \sigma$ range

$$
\tan ^{2} \theta_{12} \in[0.30,0.61]
$$

we can set lower bounds for $\left|m_{e e} / \sqrt{\Delta m_{\mathrm{ATM}}^{2}}\right|$ :

$$
\begin{array}{ll}
0.17<\left|m_{e e} / \sqrt{\Delta m_{\mathrm{ATM}}^{2}}\right| & \text { for } \mathrm{NH} \\
0.70<\left|m_{e e} / \sqrt{\Delta m_{\mathrm{ATM}}^{2}}\right| & \text { for } \mathrm{IH}
\end{array}
$$

Note that the lower bound on $\beta \beta_{0 \nu}$ for the $\mathrm{NH}$ case is especially relevant, as it is absent in the generic case. It results from this specific realization of the $A_{4}$ symmetry and is also in contrast with previous $A_{4}$-based models that led to quasi-degenerate neutrinos [19, 23].

Moreover, should future precision experiments narrow down the experimental range for $\tan ^{2} \theta_{12}$ then we might be able to distinguish between both neutrino mass hierarchies. For example, if $\tan ^{2} \theta_{12} \leq 1 / 2$ could ever be established, then we would have

$$
\begin{array}{ll}
0.23<\left|m_{e e} / \sqrt{\Delta m_{\mathrm{ATM}}^{2}}\right|<0.41 & \text { for } \mathrm{NH} \\
0.70<\left|m_{e e} / \sqrt{\Delta m_{\mathrm{ATM}}^{2}}\right| & \text { for } \mathrm{IH}
\end{array}
$$

It can also be seen that, up to order $\alpha$ corrections, $m_{e e}$ can only be zero if $\tan ^{2} \theta_{12}=1$, now strongly rejected experimentally. Note that the two solutions in Fig. 2 correspond to the two branches depicted in Fig. 1 One can see that only in the branch corresponding to the solution $b=0$ and for values of $\tan ^{2} \theta_{12}$ that are less than $1 / 2$, there is a relative minus sign between $b$ and $d$, which is the condition for inverse hierarchy, as can be seen from Eq. (3).

In the general case of complex parameters, lower bounds on $\left|m_{e e} / \sqrt{\Delta m_{\mathrm{ATM}}^{2}}\right|$ can also be established for each hierarchy. This task is simplified greatly by taking into account the reliable approximations $\sin \phi_{2} \simeq 0$, and $2|a| \cos \phi_{1}+|b| \cos \phi_{2}+|d| \simeq 0$. From Fig. 3, we see that in the complex case, lower bounds on $\left|m_{e e} / \sqrt{\Delta m_{\mathrm{ATM}}^{2}}\right|$ are indeed also established for each hierarchy. By comparing Fig. 3] with the right panel in Fig. 2] we find that these lower bounds are in fact exactly the same as obtained in the real case. The robustness of these bounds is easily understood. It follows from the fact that the maximum degree of destructive interference between the three neutrino-exchange contributions occurs in the real case with appropriate CP parities [24].

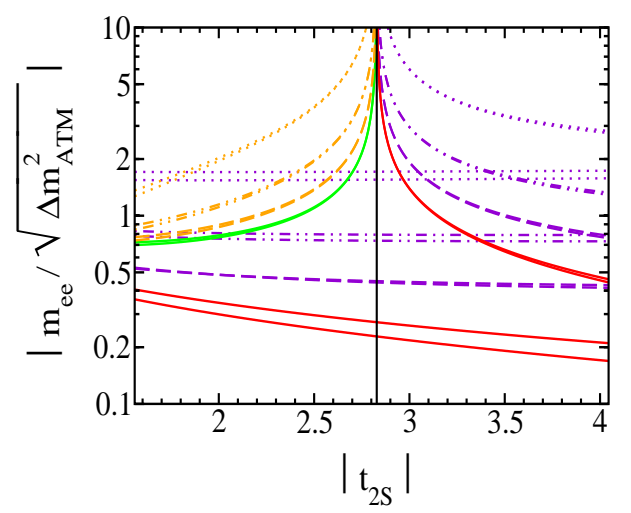

FIG. 3: $\left|m_{e e} / \sqrt{\Delta m_{\text {Aтм }}^{2}}\right|$ vs. $\left|t_{2 S}\right|$ for $\alpha=0.065$ when complex parameters are allowed.

The left-rising lines correspond to inverse hierarchy, while all others refer to the case of normal hierarchy. The lower-lying pairs of solid lines correspond to the lower bounds already discussed in Fig. [2] Again, the vertical line corresponds to $\tan ^{2} \theta_{12}=1 / 2$. Finally, the remaining lines correspond to non-zero values of the relevant $\mathrm{CP}$-violating phase varying $\cos \left(\phi_{1}\right)$ over the range $[0,1]$ in equally-spaced steps. It is conceptually interesting to note that this phase is "Majorana type" [15, 25], as we are still considering the case $\theta_{13}=0$. 
Last, but not least, we find it very interesting also that the lower bound on $\left|m_{e e} / \sqrt{\Delta m_{\text {AтM }}^{2}}\right|$ which we have obtained depends on the value of the Majorana violating phase $\left|\cos \left(\phi_{1}\right)\right|$. We can see from Fig. 4 that the lower bounds on $\left|m_{e e} / \sqrt{\Delta m_{\mathrm{ATM}}^{2}}\right|$ for each hierarchy become weaker for $\cos \left(\phi_{1}\right)=1$. Finally, we mention that,

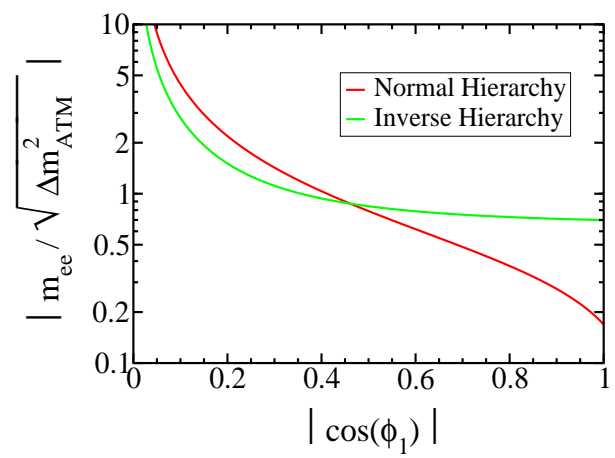

FIG. 4: Lower bound on $\left|m_{e e} / \sqrt{\Delta m_{\mathrm{ATM}}^{2}}\right|$ vs. $\left|\cos \left(\phi_{1}\right)\right|$, given current $t_{2 S}$ uncertainty. The lines in dark (red) and grey (green) correspond to normal and inverse hierarchy respectively. even though for simplicity we have focused on the case $b=c$, all results can be generalized to the case $b \neq c$, in which case $\theta_{13}$ is allowed to be nonzero.

In summary, we have given predictions for the neutrinoless double beta decay rate in a simple hierarchical variant of the $A_{4}$ family symmetry model. We showed that there is a lower bound for the $\beta \beta_{0 \nu}$ amplitude even in the case of normal hierarchical neutrino masses. We have seen that the bound is robust as it holds irrespective of whether $\mathrm{CP}$ is conserved or not. In the latter case we show explicitly how the lower bound on $\left|m_{e e}\right|$ is sensitive to the value of the Majorana phase. Our scheme suggests that neutrinoless double beta decay may be within reach of the next generation of high sensitivity experiments.

This work was supported by the Spanish grant BFM2002-00345 and by the EC Human Potential Programme RTN network MRTN-CT-2004-503369. M. H. is supported by the Ramon y Cajal programme. A. V. M. is supported by Generalitat Valenciana. The work of E.M. was supported in part by the U.S. Department of Energy under Grant No. DE-FG03-94ER40837.
[1] J. Schechter and J. W. F. Valle, Phys. Rev. D25, 2951 (1982); for a recent short review see B. Kayser, eConf C040802 (2004) L004 hep-ph/0506165.

[2] S. Hannestad, Nucl. Phys. Proc. Suppl. 145, 313 (2005), hep-ph/0412181; S. Pastor, hep-ph/0505148

[3] G. Drexlin, Nucl. Phys. Proc. Suppl. 145, 263 (2005).

[4] L. Baudis et al., Phys. Rev. Lett. 83, 41 (1999) hep-ex/9902014.

[5] C. E. Aalseth et al. [IGEX Collaboration], Phys. Rev. D65, 092007 (2002) hep-ex/0202026.

[6] H. V. Klapdor-Kleingrothaus, A. Dietz, I. V. Krivosheina and O. Chkvorets, Nucl. Instrum. Meth. A522, 371 (2004) hep-ph/0403018.

[7] I. Abt et al., hep-ex/0404039

[8] Expression of Interest in the Super-NEMO double beta decay experiment http://nemo.in2p3.fr/supernemo/

[9] R. Ardito et al., hep-ex/0501010

[10] R. Gaitskell et al. [Majorana Collaboration], nucl-ex/0311013

[11] D. Akimov et al., Nucl. Phys. Proc. Suppl. 138, 224 (2005).

[12] For an updated review of the status of global fits to neutrino oscillations and references to the recent, though already classic, experimental papers, see M. Maltoni, T. Schwetz, M. A. Tortola and J. W. F. Valle, New J. Phys. 6, 122 (2004), hep-ph/0405172.

[13] S. Weinberg, Phys. Rev. D22, 1694 (1980); E. Ma, Phys. Rev. Lett. 81, 1171 (1998), hep-ph/9805219.

[14] M. Gell-Mann, P. Ramond and R. Slansky, (1979), Print-80-0576 (CERN); T. Yanagida, (KEK lectures, 1979), ed. Sawada and Sugamoto (KEK, 1979); see also
P. Minkowski, Phys. Lett. B67, 421 (1977).

[15] J. Schechter and J. W. F. Valle, Phys. Rev. D22, 2227 (1980); Phys. Rev. D25, 774 (1982).

[16] R. N. Mohapatra and G. Senjanovic, Phys. Rev. Lett. 44, 912 (1980); Phys. Rev. D23, 165 (1981); G. Lazarides, Q. Shafi and C. Wetterich, Nucl. Phys. B181, 287 (1981).

[17] M. Hirsch and J. W. F. Valle, New J. Phys. 6, 76 (2004), hep-ph/0405015.

[18] E. Ma and G. Rajasekaran, Phys. Rev. D64, 113012 (2001), hep-ph/0106291.

[19] K. S. Babu, E. Ma and J. W. F. Valle, Phys. Lett. B552, 207 (2003), hep-ph/0206292.

[20] E. Ma, Phys. Rev. D70, 031901 (2004), hep-ph/0404199; G. Altarelli and F. Feruglio, hep-ph/0504165 E. Ma, hep-ph/0505209

[21] E. Ma and U. Sarkar, Phys. Rev. Lett. 80, 5716 (1998), hep-ph/9802445.

[22] S.-L. Chen, M. Frigerio, and E. Ma, hep-ph/0504181

[23] M. Hirsch et al, Phys. Rev. D69, 093006 (2004), hep-ph/0312265.

[24] L. Wolfenstein, Phys. Lett. B107, 77 (1981). J. Schechter and J. W. F. Valle, Phys. Rev. D24, 1883 (1981), Err. D25, 283 (1982).

[25] M. Doi, T. Kotani, H. Nishiura, K. Okuda and E. Takasugi, Phys. Lett. B102, 323 (1981); S. M. Bilenky, J. Hosek and S. T. Petcov, Phys. Lett. B94, 49 (1980); J. Schechter and J. W. F. Valle, Phys. Rev. D23, 1666 (1981); for a recent review see R. N. Mohapatra et al., hep-ph/0412099 